\title{
Erratum to: Quantifying Protein-Ligand Binding Constants Using Electrospray lonization Mass Spectrometry: A Systematic Binding Affinity Study of a Series of Hydrophobically Modified Trypsin Inhibitors
}

Dragana Cubrilovic, ${ }^{1}$ Adam Biela, ${ }^{2}$ Frank Sielaff, ${ }^{2}$ Torsten Steinmetzer, ${ }^{2}$ Gerhard Klebe, ${ }^{2}$ Renato Zenobi ${ }^{1}$

${ }^{1}$ Department of Chemistry and Applied Biosciences, ETH Zurich, 8093, Zurich, Switzerland

${ }^{2}$ Department of Pharmaceutical Chemistry, Phillipps University Marburg, 35032, Marburg, Germany

Published online: 24 April 2014

Erratum to: J. Am. Soc. Mass Spectrom. (2012) 23:1768-1777

DOI 10.1007/s13361-012-0451-6

During production of this article, an error was introduced in the formula on page 1771, which the authors did not notice when correcting the proof. The parenthesis under the big square root sign should be squared. The correct formula reads as follows:

$\frac{I(P \cdot L)}{I(P)}=\frac{1}{2}\left(-1-\frac{[P]_{0}}{K_{D}}+\frac{[L]_{0}}{K_{D}}+\sqrt{4 \frac{[L]_{0}}{K_{D}}+\left(\frac{[L]_{0}}{K_{D}}-\frac{[P]_{0}}{K_{D}}-1\right)^{2}}\right)$ 